\title{
Research of the Design of Hybrid Power Compensation Controller Based on Neural Networks
}

\author{
Zhao Jian-wei \\ Department of Computer and Information Engineering, Baoding Vocational and Technical College, \\ Baoding, China \\ 50968998@qq.com
}

Keywords: Neural networks; Hybrid power compensation; Electric filters; Harmonics compensation Abstract. The problem of harmonics compensation in power system was concerned in this paper. The method based on artificial neural network (ANN) about how to design the hybrid power compensation (HPC) controller was proposed. The HPC controller can automatically measure the harmonic currents and automatically change the compensate elements to realize the harmonic compensation. The concrete method of the ANN technology the training and test was described. The simulation results prove the validity using the ANN technology to control the HPC is an effective way to improve its dynamic performance.

\section{Introduction}

In order to suppress harmonic pollution and solve the reactive power compensation problem, the power filter and reactive compensation devices are widely applied. Many experts and product developers have made a lot of work in this regard. The traditional passive filter is bulky and its filter effect is related to the operation of system [1]. Under the specific circumstances, the resonance phenomenon may occur. In contrast, active filter can overcome the disadvantages of passive filters. It can dynamically compensate the harmonic and reactive power. And its compensation characteristics are not affected by the impedance of the power grid. However, due to limit of capacity and cost, the promotion and application of it are in trouble. In order to solve harmonic suppression and reactive power compensation, the hybrid power compensation, or called hybrid power filter, is widespread concerned. Based on the analysis of different forms of hybrid power compensation, this paper discourses the concrete method of controlling functions of ANN, and the specific methods of network design, network training and the controlling function of compensation [2].

\section{The principle and structure of HPC}

There are two main types of the HPC, one is the parallel between active power filters and LC power filters, the other is the series between active filter and LC filter [3].

The configuration of HPC consisting of static compensator and dynamic compensation based on pulse width modulation conversion is shown in Fig. 1. The part of static compensation is made by the SCR reactor static compensator and single-tuned filter group components. The static filter, according to the actual need, selects the appropriate capacitor groups specific harmonic suppression and reactive power compensation. Other harmonic suppression and reactive power compensation part harmonic are given by hybrid active power filter Thus, the structure can carry large volumes of harmonic and reactive power compensation. The PCC in the figure represents the coupling access[4]. 


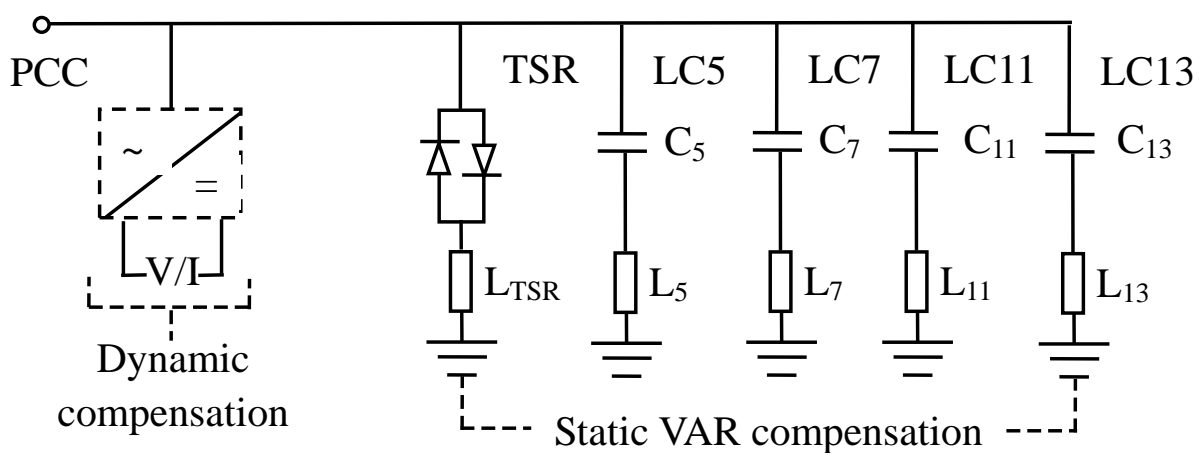

Fig. 1 Configuration of the HPC

According to the odd symmetry characteristics of most industrial load current, the odd harmonics generally need compensate, and 3rd harmonic in three-phase three-wire power supply can be ignored. Therefore, only the 5th, 7th, 11th and 13th harmonics are considered in static compensator capacitor bank. Fig. 2 shows a block diagram of connecting with single phase [5].

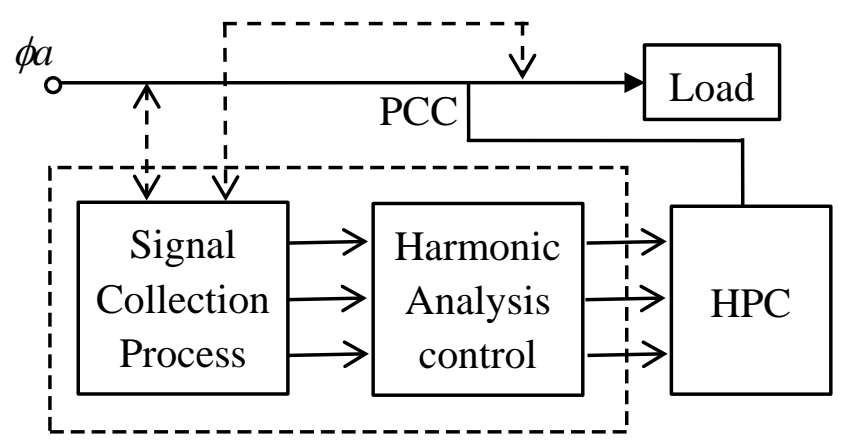

Fig. 2 Block diagram of connecting with single phase

Harmonic parameter measurement accuracy of the electric load is the key elements to ensure and enhance the hybrid power filter. The current main load harmonic measurement methods are[6]:

(1) p-q method, which is impacted by grid distortion and asymmetric voltage, and is applied to symmetrical three-phase voltage.

(2) ip-iq method, which can eliminate the effects of harmonics and voltage asymmetry, and has strong applicable ability .

The above methods can be used for analog and digital circuits, but there are some undesirable factor. Using neural networks to complete detection of harmonic currents and the hybrid power compensator control can achieve the purpose of graded compensation [7].

\section{The neural network implementation of main functions of HPC}

\section{Neural network structure design.}

In order to achieve hybrid power compensator measurement and control functions, two cascaded neural network shown in Fig. 2 approach is applied. The purpose is to reduce the complexity of the neural network, reduce training time and increase the flexibility of the system. Wherein the neural network ANN1 to achieve harmonic measurement, neural networks ANN2 to achieve harmonic control, shown in Fig. 3.

The input of ANN1 is the feature vector $\mathrm{X}$ of load current, which is obtained through the load current signal transformed by wavelet. The output are the amplitude of each harmonic signal, such 
as iQ, i5, i7, i11, i13. The output of ANN1 is selected as the input of ANN2, and the output signal of ANN2 are y1, y2, y3, y4, y5 and y6, which are the control signal of hybrid power compensation, respectively control LC5, LC7, LC11, LC13, TSR, DC filter banks, in order to achieve the harmonic current compensation control.

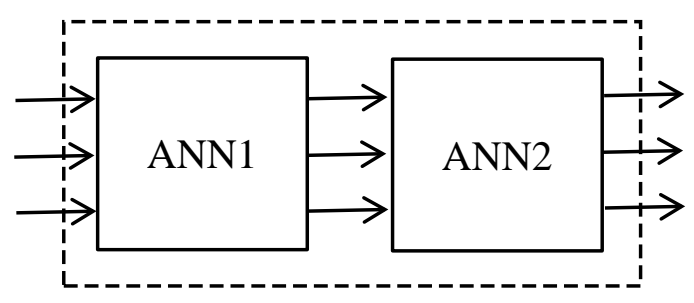

Fig. 3 Cascade of ANN1 and ANN2

The ANN1 and ANN2 both choose the three layers forward neural network, which are constituted by an input layer, a hidden layer and output layer. Moreover, the output layer of ANN1 is direcyly connected to the input layer of ANN2. The overall structure of the neural network is shown in Fig. 4.

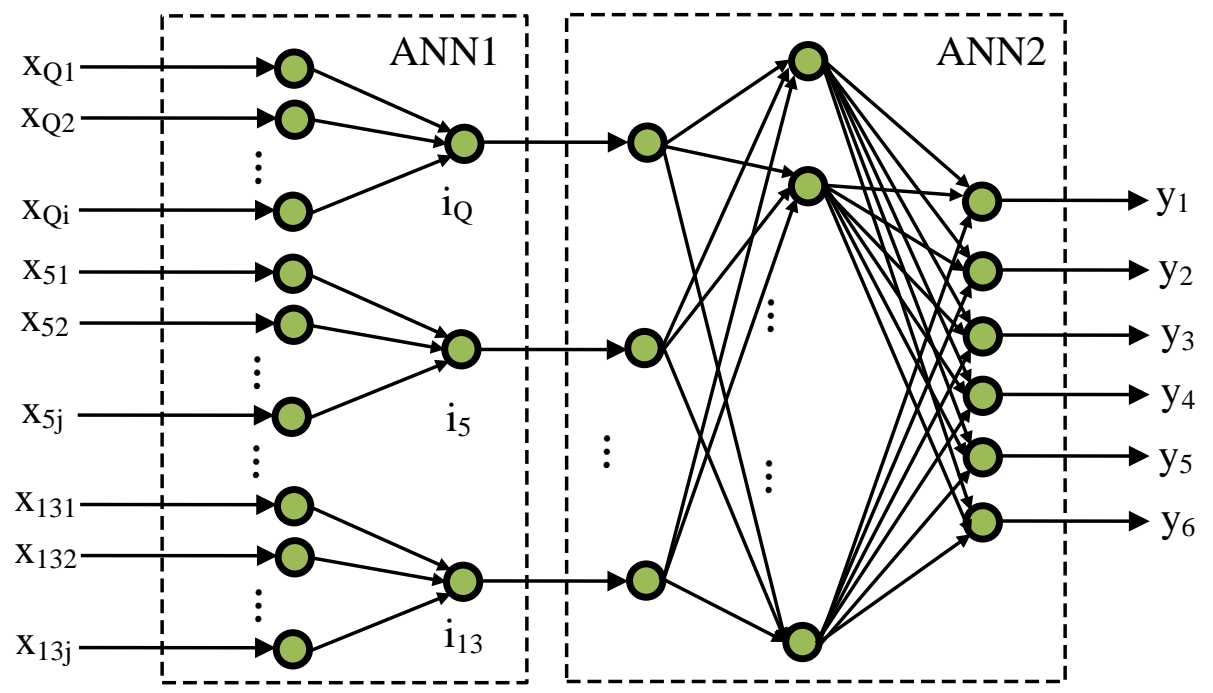

Fig. 4 ANN topology

\section{The training method of neural network.}

In order to simplify the training process, the ANN1 and ANN2 shown in Fig. 4 can be trained respectively according to theirs different functional requirements. The training of ANN1 makes complete harmonic measurement, and the training of ANN2 realizes the correct control signal of HPC filter bank corresponding to each group.

(1) The training method of ANN1

The method is carried out using MATLAB simulation. Firstly, choose a different amplitude (RMS 0-20), and contains the fundamental, 5 13 times the integer harmonics signal wavelet packet transform, extract feature vector integer values, and obtain input sample vector set and establish a target sample vector set on the hidden layer neurons using Sigmoid type transmission function, respectively, for training and simulation networks.

(2) The training method of ANN2

The training of ANN2 can still be achieved by the ANN toolbox MATLAB BP algorithm. 
Training data should be normalized based on the maximum load current. The training set and the testing set should both satisfy relative uniform distribution. Divide the input data of ANN2 into five equal intervals (Cover the entire scope of HPC). Thus, there are six possible normalization data, respectively, $0,1 / 5,2 / 5,3 / 5,4 / 5,1$, a total of 7,776 combinations of inputs, and outputs up to 64 possible working condition. Because ANN2 output neuron is 0 or 1 , the hidden layer for unipolar logarithmic function selector Sigmoid function.

\section{Simulation analysis}

Based on the simulation method described above, the neural network simulation of MATLAB toolbox is used to respectively simulate and process ANN1 and ANN2. For ANN1, the fundamental harmonic, 5th harmonic, 7th harmonics, the 11th harmonic and 13th harmonics are mainly considered. The Table. 1 and Table. 2 are respectively the simulation results of the ANN1 5th harmonics and 7th harmonics.

Table. 1 Results of simulating and testing for 5th harmonic

\begin{tabular}{|c|c|c|c|c|c|c|c|c|c|}
\hline \multirow{2}{*}{$\begin{array}{c}\text { Results } \\
\text { Input }\end{array}$} & \multicolumn{5}{|c|}{ Simulating } & \multicolumn{4}{c|}{ Testing } \\
\cline { 2 - 11 } & P51 & P52 & P53 & P54 & P55 & P56 & P57 & P58 & P59 \\
\hline RMS & 1.000 & 5.000 & 10.00 & 15.00 & 20.00 & 2.000 & 8.000 & 13.00 & 17.00 \\
\hline Target & 0.010 & 0.050 & 0.100 & 0.150 & 0.200 & 0.020 & 0.080 & 0.130 & 0.170 \\
\hline Output & 0.010 & 0.050 & 0.100 & 0.149 & 0.196 & 0.020 & 0.080 & 0.129 & 0.167 \\
\hline Absolute error & 0.000 & 0.000 & 0.000 & 0.001 & 0.004 & 0.000 & 0.000 & 0.001 & 0.003 \\
\hline
\end{tabular}

Table. 2 Results of simulating and testing for 7th harmonic

\begin{tabular}{|c|c|c|c|c|c|c|c|c|c|}
\hline \multirow{2}{*}{$\begin{array}{c}\text { Results } \\
\text { Input }\end{array}$} & \multicolumn{5}{|c|}{ Simulating } & \multicolumn{4}{c|}{ Testing } \\
\cline { 2 - 10 } & P71 & P72 & P73 & P74 & P75 & P76 & P77 & P78 & P79 \\
\hline RMS & 1.000 & 5.000 & 10.00 & 15.00 & 20.00 & 2.000 & 8.000 & 13.00 & 17.00 \\
\hline Target & 0.010 & 0.050 & 0.100 & 0.150 & 0.200 & 0.020 & 0.080 & 0.130 & 0.170 \\
\hline Output & 0.010 & 0.050 & 0.100 & 0.148 & 0.196 & 0.020 & 0.080 & 0.129 & 0.168 \\
\hline Absolute error & 0.000 & 0.000 & 0.000 & 0.002 & 0.004 & 0.000 & 0.000 & 0.001 & 0.002 \\
\hline
\end{tabular}

Table. 3 shows the simulation results of ANN2 training set, where the number of training data and test data random access to 2000 and 1000 respectively. TCR load, harmonic current range are shown as follows: i5=0 7.92A; i7=0 4A; i11=0 3A; i13=0 2.78A.

Table. 3 Simulation results of ANN2

\begin{tabular}{|c|c|c|c|c|c|c|c|c|c|c|c|}
\hline \multirow{2}{*}{$\begin{array}{c}\text { The firing } \\
\text { angle }\end{array}$} & \multicolumn{9}{|c|}{ Normalization input } & \multicolumn{7}{|c|}{ The output of ANN2 } \\
\cline { 2 - 13 } & $\mathrm{i}_{\mathrm{Q}}$ & $\mathrm{i}_{5}$ & $\mathrm{i}_{7}$ & $\mathrm{i}_{11}$ & $\mathrm{i}_{13}$ & $\mathrm{y}_{1}$ & $\mathrm{y}_{2}$ & $\mathrm{y}_{3}$ & $\mathrm{y}_{4}$ & $\mathrm{y}_{5}$ & $\mathrm{y}_{6}$ \\
\hline 0 & 0.01 & 0.91 & 1.00 & 0.92 & 0.84 & 0 & 0 & 0 & 0 & 0 & 1 \\
\hline 5 & 0.10 & 0.93 & 0.93 & 0.93 & 0.77 & 0 & 0 & 0 & 0 & 0 & 1 \\
\hline 10 & 0.17 & 0.97 & 0.71 & 0.99 & 0.63 & 0 & 0 & 0 & 0 & 0 & 1 \\
\hline 15 & 0.26 & 0.98 & 0.79 & 0.98 & 0.57 & 1 & 0 & 0 & 0 & 0 & 1 \\
\hline 20 & 0.32 & 0.96 & 0.72 & 0.90 & 0.63 & 1 & 0 & 0 & 0 & 0 & 1 \\
\hline 25 & 0.38 & 0.94 & 0.66 & 0.87 & 0.56 & 1 & 0 & 0 & 0 & 0 & 1 \\
\hline 30 & 0.43 & 0.94 & 0.56 & 0.85 & 0.46 & 1 & 0 & 0 & 0 & 0 & 1 \\
\hline 35 & 0.46 & 0.94 & 0.44 & 0.85 & 0.44 & 1 & 0 & 0 & 0 & 0 & 1 \\
\hline 40 & 0.48 & 0.92 & 0.42 & 0.78 & 0.35 & 1 & 0 & 0 & 0 & 0 & 1 \\
\hline
\end{tabular}




\begin{tabular}{|l|l|l|l|l|l|l|l|l|l|l|l|}
\hline 45 & 0.49 & 0.88 & 0.40 & 0.72 & 0.29 & 1 & 0 & 0 & 0 & 0 & 1 \\
\hline 50 & 0.49 & 0.84 & 0.41 & 0.65 & 0.25 & 1 & 0 & 0 & 0 & 0 & 1 \\
\hline 55 & 0.46 & 0.81 & 0.38 & 0.62 & 0.16 & 1 & 0 & 0 & 0 & 0 & 1 \\
\hline 60 & 0.42 & 0.77 & 0.43 & 0.55 & 0.19 & 1 & 0 & 0 & 0 & 0 & 1 \\
\hline 65 & 0.37 & 0.75 & 0.44 & 0.51 & 0.19 & 1 & 0 & 0 & 0 & 0 & 1 \\
\hline 70 & 0.33 & 0.68 & 0.55 & 0.41 & 0.28 & 1 & 0 & 0 & 0 & 0 & 1 \\
\hline 75 & 0.25 & 0.64 & 0.62 & 0.34 & 0.34 & 1 & 0 & 0 & 0 & 0 & 1 \\
\hline 80 & 0.17 & 0.58 & 0.71 & 0.25 & 0.42 & 0 & 0 & 0 & 0 & 1 & 1 \\
\hline 85 & 0.09 & 0.57 & 0.75 & 0.25 & 0.46 & 0 & 0 & 0 & 0 & 1 & 1 \\
\hline
\end{tabular}

Simulation results show that the power compensation controller based on the neural network technology is an effective way to improve the dynamic performance of the HPC.

\section{Conclusions}

Using hybrid power compensator to achieve the power system harmonic suppression and reactive power compensation is the effective method to solve the system contamination caused by grid non-linear loads. Moreover, using neural network technology for hybrid electric compensator control is the effective way to improve its dynamic performance. Simulation results demonstrate the feasibility of the proposed method. The power compensation controller based on neural network has good prospects. The initial stage of neural network power compensation controller design is based on the control program, the actual application can be made in the secondary training and practical test runs according to statistics from the scene, in order to facilitate optimum harmonic suppression and compensation effect. Therefore, it has a very good applicability.

\section{References}

[1] Zhai Qian, Lei Wanjun, Liu Tao. Development of a parallel hybrid power filter with respective harmonic compensation method. Automation of Power System. Volume 30, Issue 5 (2006), P. 49-54.

[2] Xu Yonghai, Xiao xiangning, Liu Hao. Shunt hybrid filter for harmonic suppression and reactive power compensation. Transactions of China Electrotechnical Society, Volume 20, Issue 1(2005), P. 112-118.

[3] Luo An, Fu Qing, Wang Lina. High-capacity hybrid power filter for harmonic suppression and reactive compensation in the power substation. Proceedings of CSEE, Volume 24, Issue 9 (2004), P. 115-123.

[4] Xu Wanfang, Luoan, Wang Lina. Development of hybrid active power filter using intelligent controller.Automation of Electronic Power System, Volume 27, Issue 10 (2003), P. 49-52.

[5] Fu Qing, Luo An, Wang Lina. A novel hybrid control Based on adaptive intelligent theorems for the shunt hybrid active power filter. Proceedings of CSEE, Volume 25, Issue 14 (2005), P. 46-51.

[6] Liu Xianxing, Liao Zhiling, Zhang Xinhua. Design of Digital controller for hybrid type active power filter based on DSP processor. Electrical Measurement and Instrumentation, Volume 41, Issue 459, P. 7-10.

[7] SCHOOR vanG, WYK vanJD, SHAW lans. Training and optimization of an artificial neural network controlling a hybrid power filter. IEEE Transactions on industrial electronics, Volume 50, Issue 3 (2003), P. 546-553. 